\title{
Des agriculteurs innovateurs : une nouvelle graminée dans les pâturages de Santa Maria (Amazonie brésilienne)
}

\author{
Danielle Mitja ${ }^{a}$, Pascale de Robert ${ }^{b}$ \\ a Écologue, IRD/EMBRAPA-CPAC, CP 7091 Lago Sul, 71619-970 Brasilia-DF, Brésil \\ b Anthropologue, IRD/CRBC-EHESS, 54 Bd Raspail, 75006 Paris, France
}

\begin{abstract}
Nous revenons ici sur les dynamiques à l'œuvre dans les fronts pionniers amazoniens, avec cette fois un article écrit par une écologue et une anthropologue. L'introduction d'une nouvelle graminée par des éleveurs et sa diffusion parmi les petites exploitations familiales empruntent un chemin inédit à l'écart des organismes officiels de développement. Il s'ensuit une autre configuration entre science et société, la recherche, habituellement à l'origine des innovations techniques, étant alors amenée à nouer un autre type d'alliance avec les producteurs. Cette étude de cas illustre avec pertinence la place de la technologie dans les dynamiques de développement, à l'interface entre mondes sociaux et biophysiques.
\end{abstract}

La Rédaction

\section{Mots-clés :}

innovation;

Andropogon gayanus ;

Amazonie brésilienne; agriculture familiale ; pâturages

\section{Keywords:}

innovation;

Andropogon gayanus; Brazilian Amazonia; small farmers; pastures
Résumé - L'avancée du front pionnier dans le Sud de l'État brésilien du Pará a entraîné un défrichement intensif de la forêt dense humide au profit de pâturages. Dans les petites et moyennes exploitations, ces pâturages montrent maintenant des signes de dégradation qui peuvent entraîner l'abandon de terres et la migration de familles vers d'autres lieux. Depuis 20 ans, les agriculteurs de Santa Maria ont réagi en plantant de nouvelles graminées fourragères supposées résoudre ces problèmes. L'introduction de la dernière espèce, Andropogon gayanus, est l'innovation d'un agriculteur adoptée peu à peu par tous. Elle produit une forte biomasse, résiste aux insectes et, dans un système où la pression du troupeau est faible, réussit à concurrencer les adventices. Les agriculteurs ayant donné priorité aux pâturages ont été les premiers à la tester. Le succès de l'innovation dépend de sa capacité à répondre à des problèmes donnés mais se rapporte également aux perceptions que chacun s'en fait.

\begin{abstract}
Innovative farmers: a new grass on the pastures of Santa Maria (Brazilian Amazonia). In the region of Marabá, Amazonia, the advance of the pioneer front has lead to an intensive clearing of the rainforest and to the increase of pastures that now show signs of degradation: the invasion of weeds and insect infestations. Most of the farmers perceive these problems as inevitable. However, in the last twenty years, the farmers have tried to successively introduce four fodder grasses that supposedly will solve the problem. The species most recently introduced, Andropogon gayanus, was an innovation brought by one farmer, and then progressively adopted by all of the others in Santa Maria. This type of cyclic management, where cultivated plants give satisfaction in the first time and then should be replaced by others, has quickly brought on dynamic innovations. The farmers who have prioritized pastures are constantly seeking solutions to slow down the degradation which, in the long term, implies abandoning the land and migrating with their families. Not surprisingly, they were the first to test and approve the new grass species. Those who adopted the innovation with reticence are more interested by local development alternatives that aim to ensure the sustainability of the farmers' activities (diversification, perennial cultures). The success of each innovation depends on its capacity to respond to the present problems, but also is contingent on the local perceptions of the degradation and sustainability process.
\end{abstract}

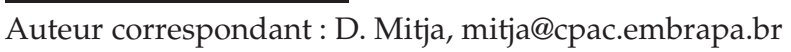


Les fronts pionniers amazoniens font l'objet d'une littérature abondante qui analyse les étapes de formation de ces nouveaux espaces gagnés sur la forêt et/ou les modes d'insertion de populations arrivées en vagues migratoires plus ou moins anciennes. La rapidité de ces processus dont on mesure encore mal les conséquences et les enjeux sociologiques, écologiques et politiques qu'ils alimentent, expliquent l'importance accordée notamment à de possibles alternatives de "développement durable $»^{1}$. Pourraient-elles contrecarrer les processus concomitants de dégradation de l'environnement, d'échappement territorial et de concentration foncière observés en Amazonie? Dans les lieux de l'après-front, soit dans les zones de colonisation ancienne, c'est le plus souvent la récupération ou la revalorisation des espaces «dégradés » et des populations « laissées pour compte » qui préoccupent chercheurs et agents de développement. Avec l'objectif de mieux connaître les raisons de la nondurabilité des systèmes ainsi que les réponses apportées par les agriculteurs eux-mêmes, nous avons abordé le thème de la dégradation/récupération des pâturages à l'échelle d'une localité du Sud de l'État du Pará.

Les colons venus s'installer dans la région de Jacundá, près de Marabá, à la fin des années 1960, trouvèrent une dense forêt humide sur les lieux où ils allaient fonder Santa Maria. Originaires de divers États du Brésil, ils pratiquent depuis lors une agriculture familiale (riz, manioc, élevage). Aujourd'hui, l'élevage extensif de bovins est la principale activité de ces agriculteurs, mais la dégradation $^{2}$ des pâturages est aussi leur principal problème. Celui-ci a entraîné l'utilisation successive de plusieurs graminées, la dernière en date étant Andropogon gayanus, innovation d'un producteur progressivement adoptée par les petits exploitants agricoles de Santa Maria. Les causes et les effets de l'introduction de cette nouvelle fourragère sont abordés avec une combinaison de points de vue à partir de la description de la végétation herbacée adventice et fourragère, de l'étude de sa dynamique, d'enquêtes sur l'histoire d'usage des pâturages

\footnotetext{
${ }^{1}$ La revue NSS, par exemple, a déjà publié plusieurs travaux sur ce thème et en particulier deux articles récents qui se rapportent à la même région d'étude que la nôtre en y apportant chacun un éclairage particulier. Albaladejo (2001) analyse les possibilités de stabilisation des populations rurales en parallèle avec le processus de mise en place de programmes de développement durable, Arnauld de Sartre (2003) évalue les chances de succès de tels projets en fonction des logiques familiales ou économiques des agriculteurs. Nous abordons cette problématique à partir de l'étude d'un cas d'innovation technique endogène dans une localité de la région.

${ }^{2}$ Du point de vue adopté ici comme de celui de l'agriculteur, un pâturage dégradé est un pâturage dont la graminée cultivée, faiblement représentée, est remplacée par des adventices herbacées et/ou ligneuses. Cette définition peut varier suivant les agriculteurs et l'usage qu'ils réservent à leur pâturage (Figuié, 2001).
}

et d'une analyse des perceptions de la dégradation du milieu. L'apport spécifique de cet article est de décrire et interpréter le succès d'Andropogon gayanus, une innovation qui n'a été recommandée par aucun organisme de recherche ou de développement.

\section{Sur le front pionnier de Marabá}

D'abord axée sur les fleuves, la région de Marabá s'est transformée en un laps de temps assez court, tant du point de vue démographique que de l'organisation du territoire, pour devenir un espace économiquement et socialement orienté par les routes construites pour servir les politiques de développement de l'État brésilien dans les années 1970 (Becker, 1990). Marabá et ses environs, autrefois couverts de forêt, ont ainsi attiré de nombreux colons qui ont participé, depuis plusieurs décennies, à la construction de nouveaux territoires et à la transformation des paysages qui sont aujourd'hui dominés par des pâturages plantés (de Reynal, 1999). Ces processus caractéristiques du front pionnier sont désormais moins actifs dans cette zone de colonisation considérée ancienne et en phase de stabilisation : les flux de population sont moins importants qu'autrefois, les terres libres sont devenues rares et les conflits fonciers relativement moins violents, de nombreuses localités sont consolidées et organisées autour d'infrastructures minimales et les agriculteurs sont partiellement intégrés au marché régional. Cependant, cette étape de la construction régionale se caractérise également par un problème bien particulier : les pâturages, qui s'étendent maintenant à perte de vue, présentent des signes de dégradation qui préoccupent agriculteurs et agents du développement. C'est sur ce constat, généralisable à d'autres régions de colonisation de l'Amazonie, que se basait le programme de recherche pluridisciplinaire dans le cadre duquel fut réalisée cette étude ${ }^{3}$.

\section{Acteurs en présence : diversité des attitudes et des contraintes}

Au-delà des quartiers urbains de la commune de Jacundá née avec la route Marabá-Belém, les grandes propriétés foncières, ou fazendas, jouxtent les terres de petits agriculteurs familiaux regroupés en «communautés », comme celle de Santa Maria (Fig. 1 et Encadré 1). Dans cette mosaïque paysagère où les interrelations sont nombreuses, chacun pratique l'agriculture et l'élevage

\footnotetext{
3 SEAH (Systèmes écologiques et actions de l'homme) du programme Environnement du CNRS : «Gestion durable des agrosystèmes en Amazonie centrale : le problème des pâturages », responsable scientifique Patrick Lavelle.
} 


\section{Encadré 1. Santa Maria, une communauté de pionniers}

Les familles qui résident aujourd'hui à Santa Maria sont presque toutes arrivées dans les années 1970 à la recherche d'une condition meilleure et occupèrent à ce moment des lots sous forêt. Les premiers temps d'installation et de lutte pour acquérir la terre ont soudé le sentiment de «communauté »; l'arrivée de nouveaux migrants est désormais rare. À partir de 1986, la route goudronnée a facilité la commercialisation de produits agricoles, comme le manioc, et notamment le lait duquel proviennent encore les rentes les plus régulières. Les agriculteurs ont investi progressivement et en priorité dans l'élevage, qui représente encore un des meilleurs moyens d'économiser (le bétail est vendu facilement en cas de besoin) et de valoriser les terres (les pâturages peuvent être vendus au fazendeiro voisin ou loués à ceux qui possèdent plus de bétail). La plupart des exploitations ont une superficie comprise entre 50 et 150 ha, certaines ont déjà épuisé leur capital forêt et se voient confrontées aux limites de la logique pionnière (Léna, 1999) : envisager une migration ou tenter de maintenir l'état de leurs pâturages en les rénovant.

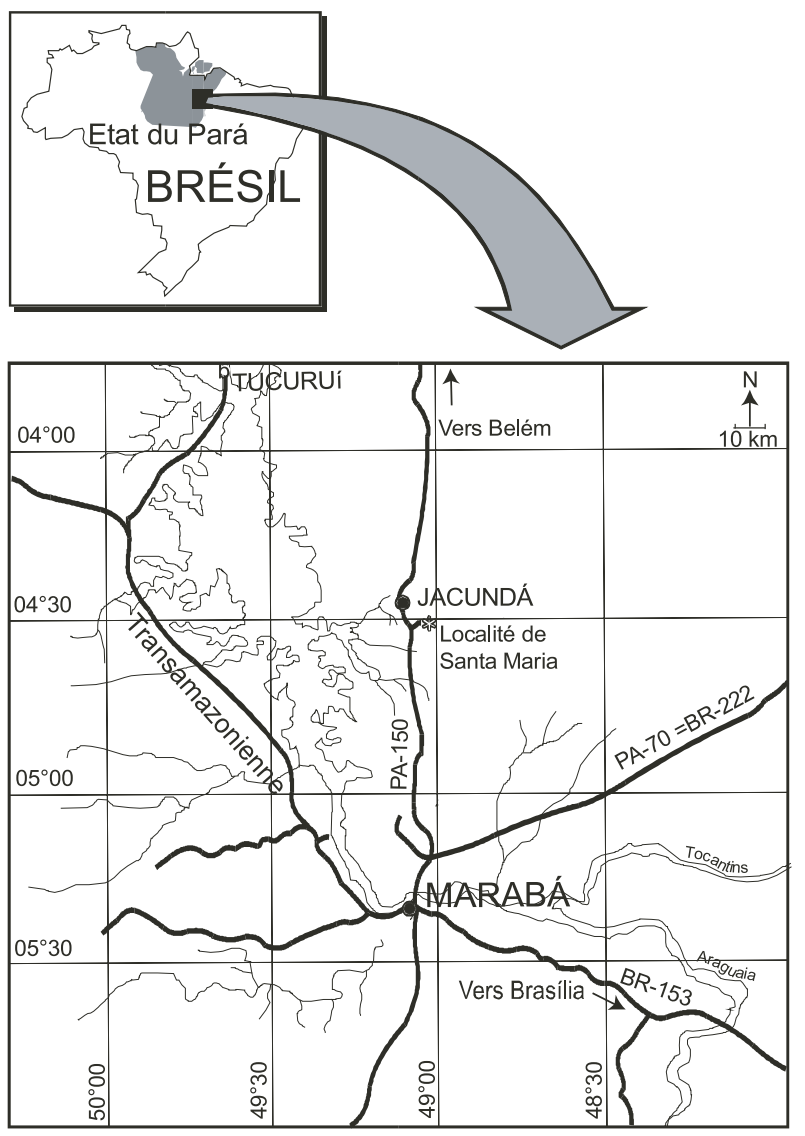

Fig. 1. Localisation du site d'étude.

avec des moyens spécifiques et différenciés, mais, en dehors de la ville, la principale activité reste l'installation et le maintien de pâturages pour l'élevage bovin.

L'agriculture familiale des fronts pionniers de la région de Marabá est pratiquée sur des exploitations relativement grandes si l'on compare avec le Sud du Brésil (Buainain et al., 2002). La taille du lot acquis à l'installation et le capital de départ sont déterminants pour l'avenir : après la coupe et le brûlis d'une parcelle de forêt dense, on cultive du riz ou du manioc, et le pâturage succède généralement à ces cultures vivrières qui doivent être installées à la place d'une autre parcelle de forêt les années suivantes (Photo 1). Les agriculteurs travaillent sans fertilisant ni machine agricole, avec une

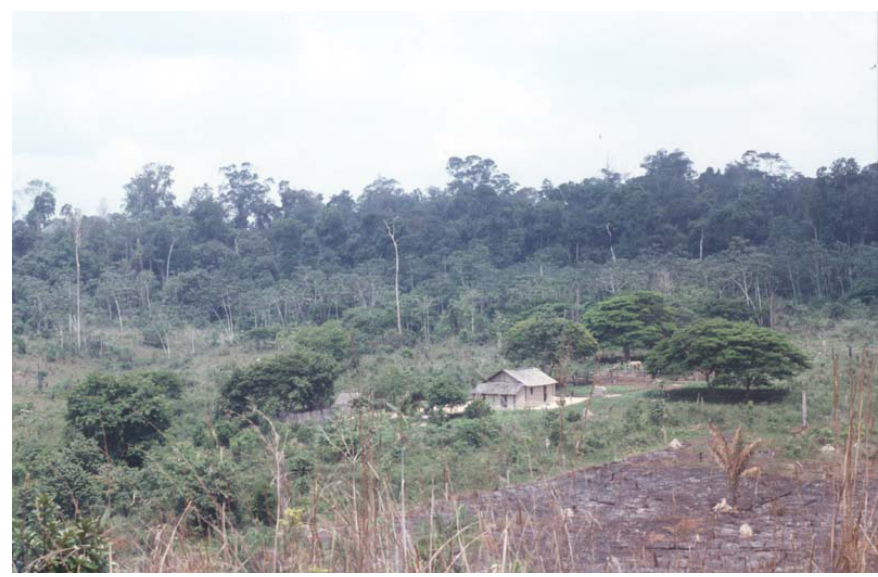

Photo 1. La végétation initiale, une forêt dense de plateau, est actuellement réduite à quelques lambeaux forestiers dispersés au milieu des pâturages. Les effets du défrichement sont plus significatifs de part et d'autre des routes et des chemins vicinaux. Dans ces paysages, les maisons d'habitation sont isolées au milieu des propriétés, les forêts s'observant en arrière-plan.

main-d'œuvre essentiellement familiale et donc limitée. La plupart d'entre eux présentent des trajectoires de vie caractérisées par une grande mobilité spatiale mais aussi sociale (voir, entre autres, de Reynal et al., 1995; Léna, 1992 ; Veiga, 1999).

Ne pouvant lutter contre la dégradation de leurs terres par manque de moyens techniques ou financiers, certains agriculteurs se voient obligés de vendre leur exploitation et de migrer plus en avant de la frontière pionnière. Ce modèle, toujours caractéristique de la région de Marabá, est toutefois loin d'être général, puisque la vente de la terre mise en pâture peut être réalisée avant la dégradation de la fourragère, laquelle dépend également des techniques et des stratégies adoptées par chaque producteur (Tourrand et al., 1995). La transaction peut aussi être assimilée à une stratégie d'accumulation servant à acquérir des moyens de production dans des trajectoires sociales ascendantes (Léna, 1992) ou alors servir des logiques familiales de rapprochement (Arnauld de Sartre, 2003). Certains tentent aussi de diversifier leur production avec, par exemple, des cultures pérennes, et de retarder ainsi les processus de dégradation (Encadré 2). 


\section{Encadré 2. Deux agriculteurs familiaux voisins et si différents}

Parmi la diversité reconnue à Santa Maria, les trajectoires de deux agriculteurs possédant des exploitations voisines se sont révélées paradigmatiques.

M. Fidelcino avait déjà une expérience d'élevage hors Amazonie et a choisi l'option « tout pâturage » en arrivant à Santa Maria. Il a rapidement défriché toute sa forêt, a installé des pâturages qu'il considère comme la culture idéale et a investi une bonne partie de son temps dans leur entretien. La superficie de son exploitation était de 115 ha. Cherchant à améliorer l'élevage et la culture de pâturage, il ne s'est pas montré intéressé par les aides à l'installation de systèmes agroforestiers proposés à l'époque par un groupe de chercheurs du LASAT/ODA, mais a suivi nos recherches sur les pâturages avec attention.

M. Placidino valorise les richesses de sa forêt (chasse, bois, etc.) qu'il a toujours défrichée avec parcimonie. Sur son exploitation de 180 ha, plus grande que celle de son voisin, il conserve des jachères qui lui permettent de cultiver périodiquement du manioc. Il a accueilli avec enthousiasme la proposition du LASAT/ODA, a monté une pépinière puis organisé une petite plantation de poivriers, orangers et autres fruitiers. Son exploitation reste dominée par les pâturages et le troupeau constitue son principal revenu, mais, comparativement à son voisin, ses pâturages sont plus envahis par une végétation ligneuse de recrûs forestiers. Il s'intéresse à d'autres alternatives de valorisation des terres.

L'introduction de nouvelles graminées peut également être une solution pour maintenir l'état des pâtures ${ }^{4}$. Rachetés par des exploitants plus capitalisés ou fazendeiros, les pâturages dégradés peuvent être rénovés et replantés, mais à la condition de disposer de tracteurs, d'engrais, d'herbicides et d'une main-d'œuvre nombreuse. . .

Entre ces deux extrêmes, on peut reconnaître toute une gamme de situations, des familles disposant par exemple de revenus externes (travail urbain, rente immobilière) et investissant avec moins de risque dans l'exploitation. L'agriculture familiale de ce front pionnier se caractérise donc par une grande diversité (Albaladejo, 2001 ; Arnauld de Sartre, 2003 ; Roy, 2002), avec des acteurs qui ont des origines, moyens et contraintes variés et qui peuvent aussi orienter leurs choix en privilégiant, par exemple, la capitalisation ou les relations sociales ou familiales.

\section{La dégradation des pâturages selon les agriculteurs}

Les agriculteurs attribuent des capacités particulières à constituer ou non un beau pâturage (pasto bonito) à chacune des espèces de graminée qu'ils sèment. Ce pâturage "propre », sans adventice, constitue l'objectif ultime de la majorité d'entre eux, mais aussi un modèle (pattern) de beauté en terme de paysage, une manifestation incontestable de la civilisation ou de la réussite sur le territoire de la forêt. Mais pourtant, une fois transformée en pâturage et souvent après le seul cycle agricole suivant la coupe de la forêt, la terre ne peut plus servir aux cultures vivrières.

\footnotetext{
${ }^{4}$ Plusieurs organismes officiels de recherche et de développement comme 1'EMBRAPA (Empresa Brasileira de Pesquisa Agropecuária), l'EMATER (Empresa de Assistência Técnica e Extensão Rural), le CAT (Centro Agro-Ambiental do Tocantins) et le LASAT (Laboratório Socio-Agronômico do Tocantins) proposent aux agriculteurs des orientations agronomiques et ont un impact important sur l'évolution de la situation agricole de la région. Le cas que nous étudions dans cet article est toutefois différent puisque la graminée Andropogon gayanus a été introduite par un agriculteur local.
}

C'est pourquoi la diminution ou la disparition, dans le lot familial, des «terres de forêt » (terra de mata) au profit des pâturages explique paradoxalement la décision de migrer de nombreux agriculteurs. Ces sols jugés fertiles assurent une indépendance alimentaire que la coupe annuelle de forêt fragilise; ils sont encore indispensables dans le système semi-itinérant en vigueur dans la région (Topall, 2001).

La prolifération des adventices et l'enfrichement qui s'ensuit préoccupent d'autant plus que ces processus peuvent mener à l'abandon pur et simple du pâturage quelques années seulement après sa mise en place. Pour qui veut porter un diagnostic sur l'état de ses terres, il est donc important d'observer les plantes et les successions végétales. Les habitants de Santa Maria nomment et classent de nombreuses plantes (souvent découvertes à leur arrivée en Amazonie) et regroupent dans la catégorie mato les adventices qui se mêlent aux fourragères semées, "salissent et prennent de la force». Alors que le terme juquira (jachère) est parfois lié à des processus de régénération dans les cycles agricoles (Veiga et Albaladejo, 2002), mato est envisagé ici comme la manifestation de la mort du pâturage ou de son abandon, c'est-à-dire de la dégradation. Il faut remarquer que celle-ci est bien souvent présentée comme inéluctable ou naturelle : le pâturage s'affaiblit peu à peu, devient «sale » et doit finalement être abandonné au mato. Ces difficultés sont souvent mises en parallèle avec d'autres, par exemple le prix du fromage, la scolarisation des enfants, la santé ou les transports. Cette vision systémique de la dégradation qui lie les questions de l'environnement à celles de la société est importante pour comprendre les manières de faire locales : les agriculteurs n'envisagent pas que le «développement » puisse se limiter à des innovations techniques restreintes à l'espace des pâturages. S'ils se démarquent ainsi de visions trop économicistes (Roy, 2002), ils accordent tout de même beaucoup de crédit aux graminées fourragères semées (capim) et comptent bien sur des orientations techniques externes pour trouver des variétés plus performantes et des solutions pour l'avenir. 
Encadré 3. L'étude d'Andropogon gayanus, résultat du dialogue entre agriculteur et chercheur

Le programme de recherche sur les facteurs de dégradation des pâturages et sur leur possible récupération prévoyait d'étudier les trois espèces les mieux représentées dans la région : P. maximum, B. humidicola et $B$. brizantha. A. gayanus, anecdotique au niveau régional, n'avait pas été retenu. C'est $\mathrm{M}$. Fidelcino qui a convaincu les chercheurs d'étudier la nouvelle graminée qu'il plantait dans une jachère herbacée en lui prédisant un énorme succès. Percevant l'intérêt de suivre le processus d'implantation d'une nouvelle fourragère, le protocole d'étude a donc été élargi. La discussion bilatérale avec les agriculteurs s'est avérée indispensable et les chercheurs sont partis du principe que les savoirs locaux et les pratiques culturales des agriculteurs devaient être pris en compte dès le début des travaux de terrain.

Le développement spectaculaire de cette espèce fourragère a donné raison par la suite aux agriculteurs qui l'avaient plantée et aux chercheurs qui avaient répondu à leur demande. Le rôle de ces derniers ne s'est pas limité à démontrer ce que les agriculteurs savaient déjà, mais il a consisté à mettre en évidence les possibles effets négatifs de cette graminée.

Les agriculteurs familiaux de Santa Maria présentent une diversité de logiques dans la gestion de leur exploitation mais partagent néanmoins le même problème, la dégradation des pâturages, et disposent de peu de moyens pour l'affronter.

\section{Une succession de graminées pour lutter contre la dégradation des pâturages}

Selon l'espace dont ils disposent, leur main-d'œuvre et leurs priorités, les agriculteurs font usage de diverses techniques pour retarder l'échéance de la friche : le brûlage annuel, l'élimination manuelle d'une partie des adventices, mais aussi la rotation des lieux de pâture, la gestion des jachères et, enfin, la recherche de graminées fourragères mieux aptes à répondre aux problèmes des pâturages.

\section{Les quatre graminées, du passé au présent}

L'histoire de l'introduction successive des graminées fourragères est bien connue des agriculteurs et des chercheurs. La première graminée semée par les fondateurs de Santa Maria était le Panicum maximum (capim colonião), mais, en quelques années, les couverts végétaux cultivés ont été envahis par des adventices herbacées et parfois par des ligneux (Serrão et Toledo, 1990; Veiga, 1995). Cette invasion, manifestation la plus visible de la dégradation, a été spontanément associée par les agriculteurs à un épuisement des terres, même si les processus de dégradation dépendent également d'autres facteurs comme la gestion du troupeau et des feux, la qualité des sols, les insectes prédateurs, etc. Bien qu'il soit toujours présent, le colonião qui a accompagné l'installation des colons est considéré localement comme l'espèce « du passé ».

Pour pallier leurs difficultés, les producteurs ont choisi de planter d'autres fourragères comme, à partir des années 1980, 1'espèce Brachiaria humidicola (capim quicuioda-Amazônia), réputée pour sa capacité à contrôler l'envahissement des adventices herbacées. Mais, depuis 1985, l'espèce $B$. humidicola s'est montrée très sensible aux attaques d'insectes qui affaiblissent les fourragères et l'espèce $P$. maximum, à port en touffes dressées, très appétée et exigeante en conduite de fertilisation, s'est également dégradée, favorisant donc dans les deux cas l'envahissement par les adventices. Une troisième espèce, Brachiaria brizantha (capim brachiarão), a été introduite en 1988. Couvrant le sol de manière presque parfaite durant les premières années de sa plantation, elle était considérée comme tolérante aux insectes parasites avant 1996, mais a subi récemment des attaques sévères dans la région de Jacundá. Des travaux actuels font aussi état, dans d'autres communes de l'État du Pará, de dommages dus à un champignon, le Pythium periilum (Teixeira et al., 2000; Veiga et Tourrand, 2001), lequel pourrait compromettre le succès que $B$. brizantha connaît actuellement dans toute l'Amazonie. Pour l'instant, le brachiarão domine largement dans le paysage régional et peut être considéré comme l'espèce du "présent ». Enfin, introduit en 1986 à Jacundá, mais diffusé plus largement dans cette commune à partir de 1991, Andropogon gayanus ${ }^{5}$ (capim andropógon) est semé ou planté, soit pour tenter de récupérer des pâturages dégradés, soit immédiatement après la culture de manioc sur des sols récemment défrichés. $C^{\prime}$ est à cette espèce du «futur » que les agriculteurs, et par conséquent nous-mêmes, avons accordé une attention particulière (Encadré 3).

\section{Caractérisation des fourragères par les agriculteurs}

À partir de leurs expériences cumulées de la culture de plusieurs graminées, les agriculteurs insistent notamment sur l'importance de la diversité des espèces,

\footnotetext{
${ }^{5}$ La graminée A. gayanus, présente dans les régions tropicales du monde entier, est originaire des savanes africaines où elle prolifère dans les jeunes jachères (Mitja, 1992). Elle a été introduite au Brésil en 1979 et le cultivar actuellement commercialisé est $A$. gayanus Kunth var. bisquamulatus (Hochst.) Hack. cv Planaltina (C.I.A.T. 621). Bien que largement diffusée dans la région des savanes brésiliennes (Thomas et al., 1981), elle n'a pas été, à l'époque, proposée aux agriculteurs d'Amazonie en raison des mauvais résultats obtenus lors des essais agronomiques (Dias-Filho et Serrão, 1980).
} 
Tableau 1. Les comportements des différentes espèces fourragères en fonction des principaux facteurs de dégradation des pâturages et des besoins du bétail ; le point de vue des producteurs.

\begin{tabular}{|c|c|c|c|c|c|c|c|}
\hline & \multicolumn{5}{|c|}{ Résistance à } & \multicolumn{2}{|c|}{ Autres qualités } \\
\hline $\begin{array}{l}\text { Capim } \\
\text { Fourragères }\end{array}$ & $\begin{array}{c}\text { Mato } \\
\text { Adventices }\end{array}$ & $\begin{array}{l}\text { Bichinhos } \\
\text { Parasites }\end{array}$ & $\begin{array}{c}\text { Seca } \\
\text { Sécheresse }\end{array}$ & $\begin{array}{c}\text { Pisoteio } \\
\text { Piétinement }\end{array}$ & $\begin{array}{c}\text { Terra fraca } \\
\text { Basse fertilité }\end{array}$ & $\begin{array}{l}\text { Production de } \\
\text { lait/viande }\end{array}$ & Appétence \\
\hline $\begin{array}{l}\text { Colonião } \\
\text { Panicum maximum }\end{array}$ & C & $\mathrm{C}-$ & C & C- & C- & $\mathrm{A} / \mathrm{A}$ & $A+$ \\
\hline $\begin{array}{l}\text { Quicuio } \\
\text { Brachiaria humidicola }\end{array}$ & $\mathrm{C}-$ & C & B & B & C & - & A \\
\hline $\begin{array}{l}\text { Brachiarão } \\
\text { Brachiaria brizantha }\end{array}$ & A & $\mathrm{B}+$ & A & A & B & $\mathrm{C} / \mathrm{C}$ & B \\
\hline $\begin{array}{l}\text { Andropógon } \\
\text { Andropogon gayanus }\end{array}$ & $A+$ & A & $A+$ & A & $A+$ & $\mathrm{C} / \mathrm{B}$ & $\mathrm{C}_{-}$ \\
\hline
\end{tabular}

Appréciations qualitatives : $\mathrm{A}=$ fort, $\mathrm{B}=$ moyen, $\mathrm{C}=$ faible.

Dans ce tableau, on n'envisage que les principaux facteurs de dégradation reconnus à la fois par les producteurs et les chercheurs : envahissement par les adventices (mato), parasites des espèces cultivées (bichinhos), sècheresse (seca), piétinement du bétail (pisoteio), basse fertilité des sols (terra fraca) et deux critères jugés essentiels pour une bonne fourragère (capim) : ses qualités nutritives et son appétence pour le bétail. (Les terminologies locales sont en italique gras.)

pour lesquelles ils reconnaissent des qualités et des limitations en fonction de leurs propres critères (Tableau 1$)^{6}$. Dans cet exercice, ils considèrent bien l'ensemble des relations qui lient les facteurs ayant une influence sur la durabilité des pâturages. Le colonião apparaît comme la fourragère la plus appréciée par le bétail et entraînant en même temps la meilleure production de lait et de viande. Malheureusement, elle est aussi exigeante en terme de fertilité des sols et sensible au piétinement, à la sécheresse, aux insectes fourragers et à l'invasion par les adventices. Les qualités attribuées au quicuio sont proches de celles décrites pour le colonião, mais il est perçu comme plus résistant à la sécheresse et au piétinement du bétail. Le brachiarão s'avère mieux adapté aux particularités et aux conditions d'élevage de la région, mais entraînerait une production en lait et viande moindre et de sérieux problèmes sanitaires chez les jeunes animaux, devant être traités avec des produits vétérinaires qui pèsent lourd dans le budget familial. L'andropógon est présenté comme la graminée la plus appropriée en raison de ses qualités et compte tenu des particularités et des limitations reconnues au milieu; elle est caractérisée comme une plante forte qui ne nécessite quasi aucun entretien et se distingue, en cela, de toutes les autres plantes domestiques. L'alimentation du troupeau par cette fourragère ne donne pas cependant d'aussi bons résultats que ceux observés avec le colonião. Bien qu'elle semble actuellement donner pleine satisfaction, les agriculteurs

\footnotetext{
${ }^{6}$ Les données proviennent de récits de vie, qui montrent les trajectoires sociales et les stratégies des agriculteurs, puis d'entretiens approfondis visant à reconnaître les catégories (de lieux, plantes, sols, etc.) et les terminologies locales nécessaires à l'étude des perceptions de la dégradation. On a cherché à analyser les relations que les agriculteurs établissent, entre autres, entre problèmes techniques, processus agroécologiques et facteurs de durabilité ou de dégradation du milieu.
}

ne s'y trompent pas : même ceux qui la considèrent maintenant comme la meilleure des solutions savent, par expérience, que des situations apparemment optimales se dégradent parfois très rapidement.

\section{Potentialités d'Andropogon gayanus, savoirs techniques et savoirs locaux}

Les caractérisations des espèces fourragères par les agriculteurs méritent d'être pondérées : alors que leurs connaissances du colonião - par ailleurs cohérentes avec les données publiées sur le sujet par les chercheurs (Peixoto et al., 1995; Diaz Filho, 1986) - se fondent sur plus de 20 ans de pratique, leurs observations de l'andropógon ne datent que de quelques années. Il apparaît donc intéressant de comparer les résultats de nos recherches (Mitja et al., 1998) et ceux de la littérature scientifique aux savoirs locaux (Tableau 1) constitués, dans ce cas, à partir d'expériences particulièrement courtes. L'étude d'Andropognon gayanus, menée par les chercheurs en raison d'un intérêt local manifeste, montre les convergences entre deux modes de savoirs et souligne en même temps la rapidité d'acquisition de connaissances sur le milieu et ses dynamiques. Mais elle met également en évidence des caractéristiques défavorables de la nouvelle graminée qui n'avaient pas été détectées par les agriculteurs de Santa Maria.

\section{Des savoirs locaux et des résultats scientifiques convergents}

Andropógon a plusieurs atouts qui le rendent attrayant pour les éleveurs. En produisant une phytomasse considérable (Photo 2), il occupe rapidement les anciens 


\section{Agriculteur 1}

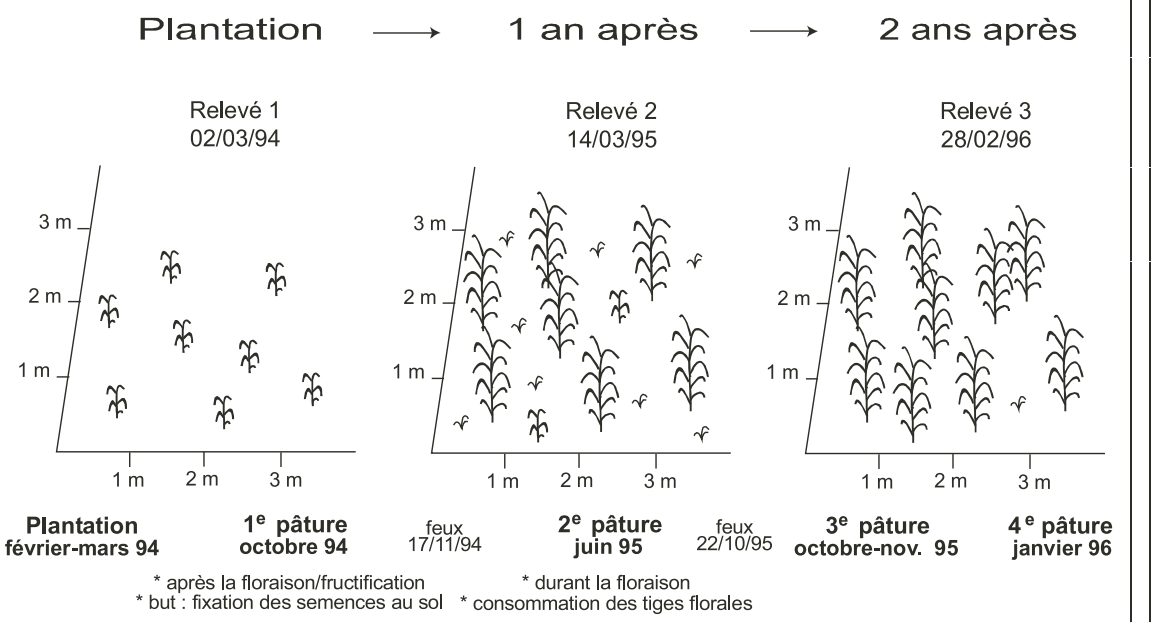

\section{Agriculteur 2}

\section{Parcelle de 5 ans}

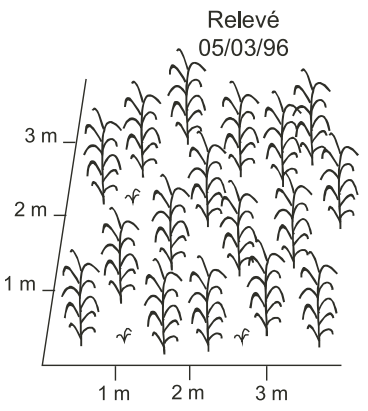

Fig. 2. Synopsis de l'étude diachronique de la parcelle 1 après sa mise en culture (durant 2 ans) et état de la parcelle 2 (5 ans). La première parcelle a fait l'objet de 3 relevés correspondant à sa plantation en boutures d'A. gayanus et à l'état de la parcelle 1 an et 2 ans après. La deuxième parcelle, mise en place par semis de graines, a été étudiée 5 ans après son installation. La présence des espèces herbacées et ligneuses adventices, l'abondance des individus, le nombre de touffes d'A. gayanus et le nombre de talles par touffe sont étudiés sur un transect de $50 \mathrm{~m}^{2}$.

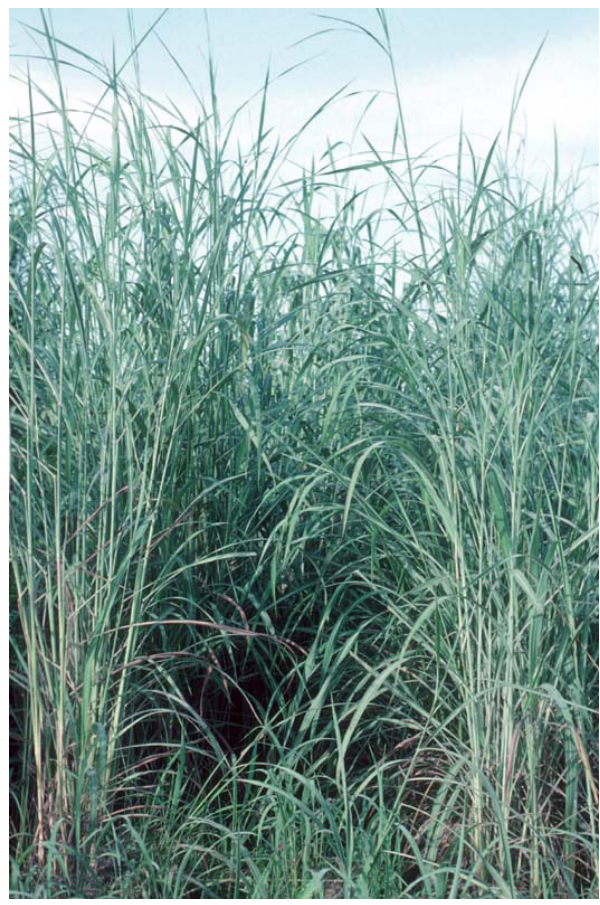

Photo 2. Touffes d'Andropogon gayanus de 2,5 à $3 \mathrm{~m}$ de haut; la forte phytomasse de la graminée assure une couverture presque complète du sol.

pâturages de $P$. maximum abandonnés après un processus de dégradation, comme nous avons pu le constater avec M. Fidelcino dans sa propre exploitation. Quelques jours seulement après le passage du feu, $A$. gayanus est la première espèce à produire de jeunes feuilles vertes. Un tel modèle de croissance est lié cependant à une gestion $\mathrm{du}$ troupeau particulièrement extensive (en moyenne une tête de bétail pour 4 à 5 ha dans la région). Cette faible charge permet d'interdire l'accès du troupeau à la parcelle d'A. gayanus durant les 6 premiers mois qui suivent la plantation, et de laisser la fourragère croître, fleurir et fructifier (Fig. 2). La première pâture n'intervient qu'après la chute des semences enfouies dans le sol par le piétinement du bétail et protégées de ce fait du passage ultérieur du feu. Elles donneront naissance l'année suivante à de jeunes touffes assurant le renouvellement du pâturage. Dès la deuxième année, les pâtures pourront être plus nombreuses, mais en revanche le nombre de plantules à la saison suivante sera moindre (Fig. 2). L'agriculteur qui est conscient de ces processus doit donc trouver un mode de gestion des terres qui permette de nourrir le troupeau tout en assurant le renouvellement périodique des pâturages. Pour cela, il utilise alternativement les diverses parcelles qui composent son exploitation.

A. gayanus concurrence efficacement les adventices herbacées (Mitja et al., 1998) en diminuant la densité d'individus des principales espèces envahissantes. Cette capacité a été parfaitement reconnue par les agriculteurs de Santa Maria, qui caractérisent la fourragère plantée andropógon avec des qualités normalement réservées aux seules adventices invasoras («envahisseuses ») ou mato. La graminée Andropogon gayanus permet donc de récupérer des pâturages envahis par les adventices, ce qui constitue un avantage certain par rapport aux autres fourragères et explique son succès; surtout chez les petits agriculteurs qui n'ont pas d'autres moyens pour rénover leurs pâturages. Pour concurrencer les adventices, 


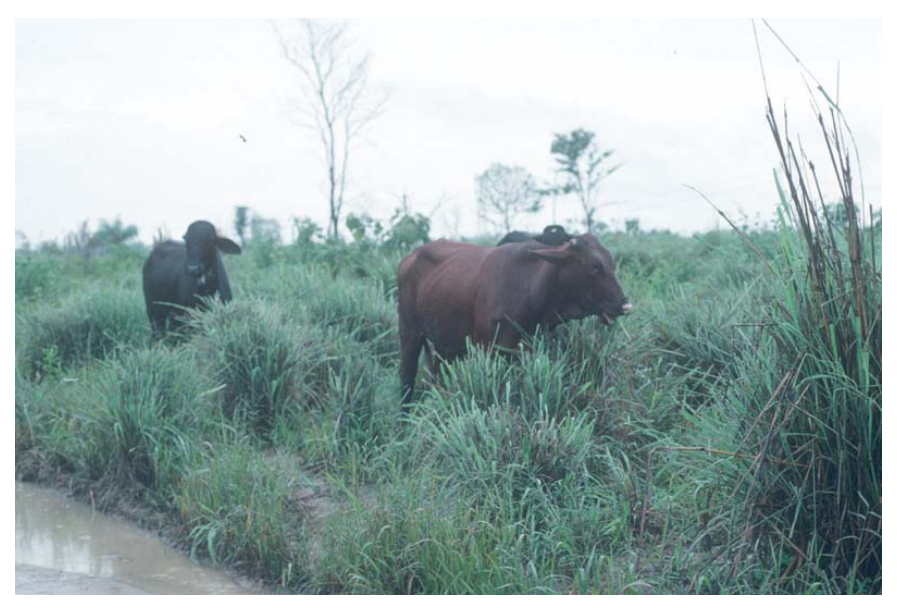

Photo 3. Vaches broutant Andropogon gayanus.

la graminée $A$. gayanus adopte deux stratégies : elle occupe l'espace grâce à ses grosses touffes et produit des semences viables qui permettent un renouvellement permanent du pâturage.

Pendant la saison sèche, $A$. gayanus conserve des feuilles vertes qu'il renouvelle à la moindre pluie (Mejía, 1984) et, bien que cette masse verte soit peu abondante, elle peut assurer un minimum de fourrage pour le bétail au moment où les autres fourragères cultivées sont sèches. C'est en ce sens que les agriculteurs qualifient andropógon de fourragère résistante à la sècheresse.

La forte production d'A. gayanus est constatée par les agriculteurs comme par les chercheurs, même en condition de faible fertilité (Mitja et al., 1998) et elle est stable, même sans addition d'engrais (Thomas et Andrade, 1984). Ceci la rend encore plus intéressante pour les producteurs amazoniens qui disposent de ressources limitées et n'utilisent aucun intrant chimique. Comme ils le disent eux-mêmes, «andropógon aime les terres faibles (terras fracas) ».

A Santa Maria, A. gayanus était inconnu des vaches, qui ont eu quelques réticences à le consommer. Ce défaut a été signalé de manière récurrente par les éleveurs. Pourtant, avec le temps, l'acceptation de la plante par le bétail se révèle satisfaisante ${ }^{7}$ (Photo 3 ). La valeur nutritive d'A. gayanus, comme celle de la plupart des espèces fourragères, diminue avec l'augmentation de l'âge de la repousse. Elle est considérée comme modérée selon sa valeur de digestibilité et sa composition chimique (Jones, 1979), mais les fortes productions de phytomasse compensent cet inconvénient. Leite et Zoby (1999) ont montré, dans la région des Cerrados, un gain de poids supérieur pour le bétail qui consomme A. gayanus par

\footnotetext{
${ }^{7}$ En général, $A$. gayanus a la réputation d'être bien accepté par le bétail. Dans la région des Cerrados brésiliens, Thomas et al. (1981) ont montré que lorsque les animaux ont un libre accès à 5 graminées fourragères, la préférence va à $A$. gayanus.
}

rapport à celui qui consomme $B$. brizantha, fait que les agriculteurs ont également perçu.

Enfin, l'espèce $A$. gayanus est actuellement la graminée fourragère qui résiste le mieux à l'insecte cigarrinhadas-pastagens $^{8}$ (Cosenza et al., 1989). Cette qualité, une des premières à être mise en valeur par les agriculteurs, est incontestablement à l'origine de sa réussite dans la localité.

\section{Des résultats que les agriculteurs ne soupçonnaient pas}

Par leur pratique, les agriculteurs ont accès à un mode de connaissance des processus agroécologiques que l'approche scientifique se contente souvent de confirmer : la plupart des qualités de la nouvelle fourragère avaient déjà été reconnues par les agriculteurs. Sur un certain nombre de points, toutefois, les résultats scientifiques permettent en quelque sorte d'anticiper sur la connaissance empirique en reconnaissant des caractéristiques d'Andropogon gayanus qui sont, pour l'instant, difficiles à percevoir.

Alors que globalement la consommation d'A. gayanus entraîne un gain de poids supérieur pour le bétail, il existe une variation saisonnière différente suivant la fourragère. Ainsi $B$. brizantha occasionne un gain de poids supérieur en saison sèche, alors que $A$. gayanus entraîne une plus forte croissance des animaux en saison des pluies (Saraiva et Barcellos, 1996). Cette variation saisonnière n'est pas facile à évaluer dans une exploitation, car la croissance du troupeau dépend de nombreux autres facteurs (race des vaches, disponibilité en sels minéraux, rotation des parcelles pâturées... ).

L'étude d'A. gayanus (Mitja et al., 1998) a également permis de mettre en évidence un risque potentiel : l'installation de la fourragère occasionne une diminution de la densité des adventices mais n'influence pas la richesse spécifique, qui reste constante. En d'autres termes, si la gestion du troupeau venait à changer et que sa pression sur le pâturage augmentait de manière significative, une nouvelle prolifération des adventices, toujours présentes, serait à craindre.

Un autre inconvénient potentiel est constitué par les grosses tiges florifères $\mathrm{d}^{\prime} A$. gayanus qui commencent à se dessécher après la production des semences. Ce matériel persistant, difficilement dégradable, limite l'accès du bétail aux feuilles nouvelles pourtant produites tout au long de l'année. Dans un contexte de feux annuels, cet inconvénient est périodiquement résolu. Cependant, la tendance actuelle, renforcée depuis l'implantation massive

\footnotetext{
8 Insecte homoptère représenté surtout par l'espèce Deois incompleta.
} 


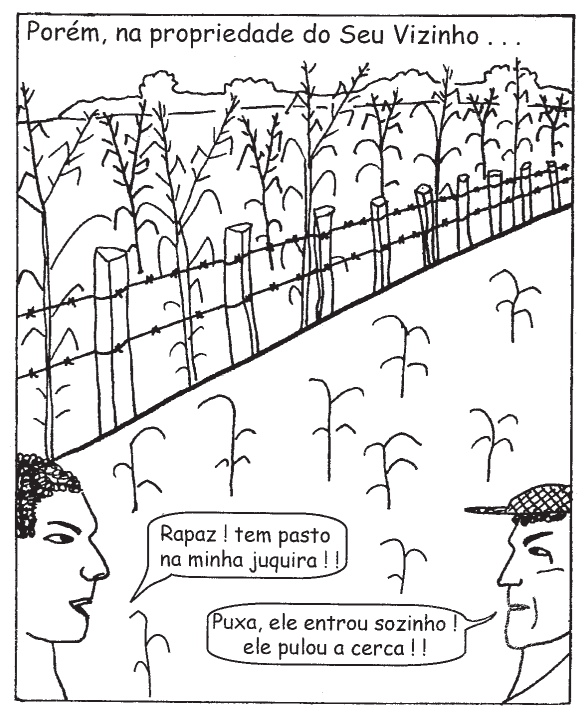

Dessin 1. Envahissement de la jachère à droite de la clôture à partir de semences provenant d'Andropogon gayanus du pâturage situé à gauche de la clôture (Mitja, 2004).

de $B$. brizantha - une espèce sensible au feu - est à la diminution, voire à la suppression des feux systématiques ${ }^{9}$.

La viabilité des semences $\mathrm{d}^{\prime} A$. gayanus, considérée par les chercheurs et les agriculteurs comme un avantage lorsqu'elles tombent dans l'enceinte de la parcelle, peut devenir un inconvénient majeur quand ces mêmes semences, emportées par le vent, viennent à franchir la barrière du champ et à s'installer dans la parcelle du voi$\sin ($ Dessin 1). De proche en proche, on pourrait assister à une invasion par cette fourragère qui risquerait alors de concurrencer les autres espèces plantées, et même de les éliminer (Klink, 1996). Une telle perspective de monoculture fourragère pourrait être dramatique pour le futur de la région. Alors que les agriculteurs ont bien assimilé andropógon à une plante du mato en lui conférant des propriétés caractéristiques de plantes sauvages comme les adventices, aucun n'avait envisagé que cette graminée pourrait un jour envahir à son tour l'écosystème et compromettre leurs activités.

En conclusion, le succès d'A. gayanus est lié au fait qu'il possède un ensemble de caractéristiques agronomiques et écologiques intéressantes pour une certaine catégorie d'agriculteurs, à un moment donné de l'histoire de leur exploitation. Une espèce qui résiste aux insectes parasites et produit une forte biomasse pour nourrir le troupeau, tout en limitant les investissements en main-d'œuvre ou herbicides contre les adventices et en médicaments pour le bétail, présente incontestablement

\footnotetext{
${ }^{9}$ La pratique du brûlage est maintenant très sérieusement limitée par la loi. Des recherches entreprises à l'EMBRAPA, pour tenter de résoudre ces problèmes spécifiques de l'espèce A. gayanus, ont abouti à la sélection de la variété «baetí » à tiges florales plus fragiles (Leite et al., 2001).
}

de grands atouts pour les agriculteurs familiaux ${ }^{10}$. Les risques associés à andropógon sont d'autant plus minimisés par les agriculteurs qu'ils maintiennent une diversité des pâturages (quatre espèces fourragères plantées). En revanche, si $A$. gayanus avait été introduite au moment de l'apogée du colonião, elle aurait probablement été délaissée par les agriculteurs qui regrettent encore l'exceptionnelle graminée « du passé ».

\section{La circulation et l'appropriation de l'innovation : regarder, parler, planter}

La mobilité des agriculteurs familiaux favorise la circulation d'informations et d'innovations à travers les réseaux sociaux alors que la diversité de leurs origines, expériences et savoirs semble faciliter les processus d'appropriation des nouvelles techniques et connaissances. L'innovation, par définition une prise de risque, est bien ici une activité «ordinaire » mais elle n'apparaît pas pour autant une forme de "déviance» ou de "transgression » (Alter, 2000) probablement parce que la norme - au moins technique - n'est pas vraiment établie : les systèmes de pratiques institués sont toujours actuellement «travaillés » aussi bien de l'extérieur que de l'intérieur, comme le montre le cas étudié. D'autre part, dans la mesure où elle ne remet pas en cause les choix déjà effectués par les autres, l'innovation ne s'inscrit pas nécessairement dans des rapports de pouvoir au sein de la localité, où dominent ici de toute manière les relations horizontales.

\section{Innover c'est leur vie}

La «frontière » constitue un territoire en formation où les évolutions s'avèrent très rapides et souvent radicales. Sur deux décennies, par exemple, les habitants de Santa Maria perçoivent des changements significatifs dans l'intensité et la répartition des pluies. Mais le milieu naturel n'est pas seul à se transformer : le nombre d'habitants de la localité, les réseaux de voisinage, les services sanitaires et sociaux, les conditions d'accès à un marché fluctuant, et même les toponymies ou les rêves familiaux évoluent sans cesse. La nouveauté, l'imprévu, le changement font partie du quotidien et l'innovation ne répond pas seulement, bien évidemment, aux problèmes posés par la dégradation des pâturages. Des initiatives comparables à l'innovation andropógon ont déjà été mises en évidence

\footnotetext{
${ }^{10}$ La plupart des fazendeiros disposent de produits vétérinaires et phytosanitaires et peuvent rénover mécaniquement leurs pâturages qui sont le plus souvent monospécifiques et généralement plantés en brachiarão. Cependant, certains d'entre eux s'intéressent aussi à A. gayanus, comme par exemple M. Mauro (Encadré 4).
} 
Encadré 4. La diffusion de la semence d'andropógon

En 1986, M. Mauro, propriétaire d'une exploitation de 2100 ha, s'est rendu dans le Goiás à plus de 1300 km de sa résidence. De ce voyage dans la région des Cerrados du Brésil, il a ramené des semences d'A. gayanus et les a semées dans une de ses parcelles de Jacundá. Cette espèce se développant bien, il a décidé de la planter sur une grande surface et d'importer des semences pour les vendre. Ce n'est que 5 ans après son introduction à Jacundá qu'A. gayanus sera disponible sur le marché local (Dessin 2) et effectivement acheté par deux autres agriculteurs qualifiés de fazendeiros : M. Claudionor, qui possède 426 ha à Santa Maria et exerce une profession libérale à Jacundá, et M. Jeremias, qui aurait probablement vendu son exploitation de 1800 ha au sud de Jacundá sans cette nouvelle option de pâturage.

Il faudra encore attendre 2 ans pour que la fourragère s'impose dans les pâturages des petits agriculteurs de Santa Maria. M. Fidelcino a essayé le premier cette espèce, en 1993, sur une petite superficie de son exploitation après l'avoir vue croître chez M. Claudionor qui lui en vante aussi les mérites. Ses voisins, M. Sebastião et M. Placidino, firent de même mais après avoir observé un à deux cycles de la graminée sur les terres de M. Fidelcino, véritable «pionnier » de l'andropógon chez les petits agriculteurs de la localité, avec lesquels il dialogue par ailleurs régulièrement. Puis tous les agriculteurs de Santa Maria furent semblablement convaincus des aptitudes de la nouvelle fourragère, désormais plantée partout. Par le biais d'une réunion de restitution des résultats, organisée par le LASAT, à Marabá en 1999, les agriculteurs de Santa Maria ont rencontré ceux de localités voisines. Les résultats de la recherche exposés, le débat s'est engagé entre les agriculteurs qui avaient une expérience d'A. gayanus et qui ont souvent pris la parole pour appuyer ou compléter les dires des chercheurs (Photo 4) et ceux qui ne le connaissaient pas. Plusieurs parmi ces derniers se sont montrés intéressés lors de la réunion et deux ans plus tard, en 2001, nous avons pu observer de jeunes plants d'andropógon dans les pâturages d'autres localités, comme Pau seco située à $85 \mathrm{~km}$ de Santa Maria. Suite à la réunion, qui regroupait une cinquantaine d'agriculteurs, et en vue d'une diffusion plus large des résultats immédiatement applicables de la recherche, une bande dessinée présentant les avantages et les inconvénients de l'introduction d'A. gayanus a été élaborée (Mitja, 2004). Les dessins 1 et 2 font partie de ce document. Celui-ci a été distribué en janvier 2004 lors d'un séminaire réalisé à Marabá par le LASAT et destiné aux syndicats d'agriculteurs.

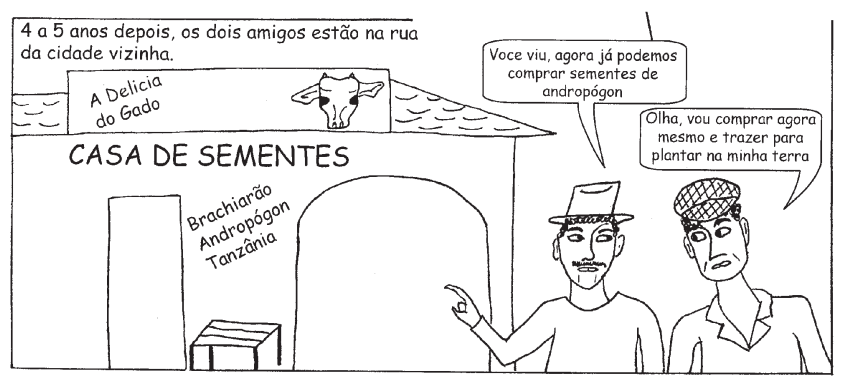

Dessin 2. Deux agriculteurs discutant devant le magasin où ils achètent les semences de fourragères (Mitja, 2004).

dans d'autres secteurs agricoles, mais aussi dans d'autres domaines de la vie sociale : culture et commercialisation du roucou (Bixa orellana), création d'associations pour des projets collectifs ou expériences isolées de gestion forestière, d'apiculture, etc. (Veiga, 1999; Assis, 2001).

Dans un contexte dynamique où le milieu naturel se transforme rapidement, les normes techniques sont l'objet d'un ajustement continu au fur et à mesure que les connaissances du milieu se développent et que des nouveautés sont introduites dans les façons de faire (Darré, 1996). La pression de l'environnement agroécologique en voie de dégradation stimule incontestablement cette course à l'innovation et, dans ces conditions, l'adoption de la nouvelle graminée pourrait être caractérisée comme une stratégie de type «défensif » (Yung et al., 1995). Sur le front pionnier, l'innovation technique apparaît bien alors comme une pratique ordinaire, voire une obligation, indispensable à la survie même des exploitations et à la permanence des populations rurales. Ainsi, l'introduction de

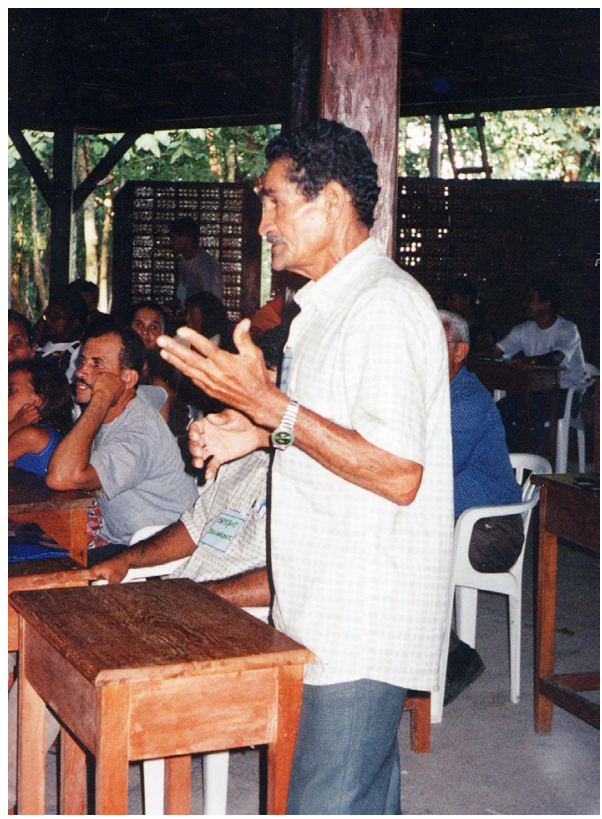

Photo 4. M. Placidino prenant la parole lors de la réunion d'agriculteurs de mars 1999 au cours de laquelle ont été présentés les résultats de la recherche.

nouveaux modes d'exploitation, la transformation des gestes techniques, la diffusion et l'adoption d'innovations intéressent très fortement les agriculteurs de Santa Maria et jouent pour eux un rôle essentiel. Enfin, la diversité des agriculteurs, d'abord tous «étrangers » à la localité de par leurs origines, et porteurs chacun d'expériences singulières, augmente encore la capacité du groupe à innover. 


\section{Les réseaux d'échange}

Les agriculteurs sont arrivés à Santa Maria avec un bagage technique donné : souvent, ils avaient déjà acquis une certaine expérience de l'élevage, mais hors du milieu amazonien. L'apprentissage de nouvelles manières de faire est une étape essentielle à l'installation, sans doute déterminante par la suite pour la survie et la longévité de l'exploitation. Regarder les pâturages et les troupeaux des autres, parler à son voisin, travailler régulièrement avec lui et chercher à s'informer toujours mieux, sont ici d'indispensables préalables à l'action. Les conditions d'insertion sociale dans la localité sont de ce point de vue importantes et se mesurent à la qualité des relations de voisinage qui peuvent être établies à l'arrivée, à la densité des relations de parenté retrouvées ou reconstruites sur place (Araújo, 1996). Les agriculteurs familiaux, desquels on a déjà souligné la mobilité, maintiennent également des liens à l'extérieur, sur les lieux où ils ont vécu, ceux dont ils sont originaires, ou avec des parents installés ailleurs. Les fréquents voyages hors de la localité, pour des visites ou des travaux saisonniers par exemple, sont encore d'autres occasions mises à profit pour s'informer de pratiques nouvelles. De plus, les semences utilisées pour l'implantation des pâturages sont achetées et très rarement cultivées ou échangées. Le magasin de semences, en ville, est donc également un lieu de dialogue, d'échange d'expériences et de diffusion de nouvelles espèces. Dans la région de Marabá, il existe par ailleurs un réseau actif d'institutions qui s'intéressent de diverses manières aux agriculteurs ${ }^{11}$, en proposant des formations, en soutenant des actions politiques ou des projets de développement. Ceux-ci disposent donc d'une certaine habitude du partenariat et font preuve de confiance vis-à-vis du progrès agronomique, comme le montre, par exemple, l'intérêt porté à nos recherches ou aux réunions de divulgation ayant pour objectif de transmettre des connaissances utiles à la gestion des pâturages. Chaque agriculteur participe donc de différentes manières à des réseaux locaux et extra-locaux qui se caractérisent par des liens plus ou moins forts, ce qui est supposé favoriser, au moins, la circulation de l'information technique.

\section{Caractéristiques de l'innovation andropógon}

On peut considérer que l'andropógon s'est diffusé à travers trois réseaux superposés, celui des grands

\footnotetext{
${ }_{11}$ Notamment le LASAT relié au NEAF/CAP/UFPa (Núcleo de Estudos Integrados sobre Agricultural Familiar / Centro Agropecuário/Universidade Federal do Pará) qui travaille depuis longtemps sur la question de la durabilité des pâturages et de la viabilité des exploitations, participe à des formations professionnelles pour les agriculteurs de la région et organise, avec eux et/ou les syndicats qui les représentent, des séminaires où sont restitués et discutés les résultats des recherches.
}

propriétaires qui partagent les mêmes intérêts, celui des agriculteurs de la localité qui maintiennent entre eux des liens forts et le réseau élargi constitué à partir des actions des organismes travaillant pour le développement local. Comme on l'a vu, A. gayanus a été introduit par un agriculteur, qui a pris seul le risque de l'innovation (Encadré 4). À l'inverse des alternatives techniques venant de programmes de développement, ce type d'innovation spontanée ou endogène est moins couramment étudié (Veiga, 1999; Chauveau, 1995), mais s'avère correspondre souvent aux expériences ayant abouti à un réel succès. Comme cela a déjà été observé au Brésil, l'innovateur est ici un propriétaire aisé (Rogers, 1983). Il était aussi à l'affût de solutions pour son exploitation en voie de dégradation et avait la possibilité matérielle de les réaliser. L'andropógon a donc été importé, testé puis conseillé par M. Mauro et, finalement, acheté, testé et adopté par M. Jeremias et M. Claudionor. Dans cette innovation «par imitation» (Mollard, 1999), un individu introduit une nouvelle espèce à titre expérimental. Le succès de l'implantation se manifeste à travers l'adoption de la plante par d'autres agriculteurs disposant eux aussi d'une certaine capacité de réponse au risque, avant de s'imposer progressivement chez ceux dont les ressources sociales et techniques sont moindres. D'expérience marginale menée par un propriétaire disposant d'une plus grande marge de manœuvre quant au risque agricole, A. gayanus est progressivement réapproprié par une majorité d'agriculteurs.

Innovateur ${ }^{12}$ dans la communauté de Santa Maria, M. Fidelcino s'est d'abord convaincu des résultats obtenus chez les fazendeiros avant de tester andropógon chez lui sur une petite superficie. Il plante ensuite la fourragère à plus grande échelle, observe quelque temps son évolution en fonction de la pression de pâture puis adapte ses techniques selon un processus de réappropriation de l'innovation (Darré, 1996; Albaladejo, 2001) dont nous avons détaillé le résultat, pour ce cas concret, sur la Figure 2. Comme le rappelle Alter (2000, p. 69), toute appropriation suppose une création de sens : la semence avait été introduite sans le bagage explicatif et technique venant habituellement avec les nouvelles cultures proposées de l'extérieur - sans mode d'emploi en quelque sorte - si ce n'est celui des fazendeiros, en partie inadapté (ils disposent de moyens différents pour soigner leurs pâturages). C'est donc M. Fidelcino qui a «inventé » les modalités de culture de l'andropógon adaptées, en gestes et en mots, à l'agriculture familiale de l'endroit. De cette manière, 1 'innovation endogène est bien intégrée au système local de compréhension des pâturages et ainsi,

\footnotetext{
${ }^{12}$ L'innovation suppose une prise de risque, mais M. Fidelcino, contrairement à M. Mauro, ne disposait pas de plus de ressources que ses voisins en cas d'échec. Au contraire, son initiative s'explique par un choix initial donnant priorité aux pâturages sur son exploitation.
} 
pour les agriculteurs, elle « fait sens » dès le début. C'est pour cette raison notamment qu'elle a des chances de succès et qu'elle est finalement appropriée par tout le monde.

\section{Des logiques différenciées face à l'innovation fourragère}

Comme nous l'avons déjà souligné, dès qu'une nouvelle graminée intéressante est disponible, diverses attitudes s'observent parmi les producteurs de Santa Maria. Certains sont prêts à se lancer immédiatement dans l'aventure ou après des observations chez le voisin et des expérimentations sur des parcelles réduites, d'autres se montrent moins enthousiastes, même s'ils finissent par adopter l'innovation. Plutôt que d'être évaluée sur ses seuls résultats, la plante fourragère est regardée en fonction du système technique qu'elle sous-tend, et auquel on adhère plus ou moins. Ceux qui semblent y résister rêvent encore du temps révolu du colonião, refusent ou retardent la coupe de leur forêt, garante des futures récoltes de riz et de manioc, et craignent encore l'objectif «tout pâturage » choisi pourtant par la majorité et, de ce fait peut-être, difficile à éviter à Santa Maria. À l'instar de M. Placidino qui a tardé à adopter la nouvelle graminée (Encadré 2), ils montrent par ailleurs des stratégies un peu différenciées : souvent plus attirés par la diversification des activités, ils peuvent chercher à mieux valoriser la surface de forêt dont ils disposent encore (en limitant les coupes, en vendant mieux leur bois), entretenir plus de terres agricoles (pour la consommation familiale ou la vente) ou orienter leurs enfants pour une insertion locale durable (sur l'exploitation ou/et à Jacundá). S'ils ne se trouvent pas toujours à l'aise dans le contexte des fortes dynamiques actuelles, ces agriculteurs se montreront certainement innovateurs dans un système garantissant une plus grande longévité aux exploitations. Ainsi, tout en manifestant clairement leur désir de rester sur place et de rompre avec les cycles de migrations, ils montrent de l'intérêt, par exemple, pour les cultures pérennes et les systèmes agroforestiers ou font preuve d'initiatives pionnières (apprivoisement $\mathrm{d}$ 'animaux sauvages).

Même si chacun a des aptitudes à innover ou à accepter plus ou moins rapidement une innovation et que l'on puisse regrouper les individus suivant ces critères (Rogers, 1983), il faut encore considérer les différences de comportements en fonction du type d'innovation proposé et de son contexte d'implantation. Sans chercher à établir une typologie à partir du cas étudié, on peut quand même caractériser M. Fidelcino comme un innovateur précoce pour l'Andropogon gayanus alors qu'il n'a pas montré d'intérêt pour les innovations de type cultures pérennes qui passionnaient au contraire son voi$\sin$, M. Placidino. La recherche, l'acceptation rapide, lente ou le refus d'une innovation dépendent de la manière dont chaque agriculteur envisage, en fonction de ses propres logiques, l'évolution de l'exploitation et, d'une manière générale, "le développement ». L'influence locale des agriculteurs qui se montrent innovateurs dans un système tout pâturage ou, au contraire, innovateurs dans un système stable plus diversifié joue sans doute un rôle important pour le succès ou l'échec de tel ou tel projet de développement à l'échelle de la localité. Par ailleurs, les innovations endogènes qui se diffusent au sein de groupes où dominent les relations horizontales (comme c'est le cas à Santa Maria où les agriculteurs disposent aujourd'hui de moyens comparables) et les liens forts pourraient avoir moins d'effets sur les processus de différenciation sociale que celles, exogènes, suscitées par des agents externes et diffusées à partir d'autres réseaux moins locaux. Le cas andropógon étudié pourrait du moins illustrer une telle hypothèse.

\section{Conclusion}

Si l'innovation que constitue l'introduction de la graminée $A$. gayanus s'avère être un succès, c'est d'abord - au-delà de ses propres qualités - parce qu'elle est arrivée au bon moment à Santa Maria. Les autres espèces fourragères, pourtant très satisfaisantes peu auparavant, étaient affectées par la cigarrinha ou laissaient les parcelles salies par le mato. A. gayanus, "qui se comporte comme du mato ", a permis de récupérer des pâturages considérés dégradés, voire irrécupérables, avec les seuls moyens dont disposent les agriculteurs et de leur propre initiative. Andropogon, si apprécié dans les conditions agroécologiques actuelles, n'aurait sans doute pas remporté une telle adhésion s'il avait été introduit quelques années plus tôt, lors de l'apogée du P. maximum. Le succès d' andropógon, comme celui de toute innovation, dépend donc autant de ses capacités agroécologiques que du contexte socioéconomique de son introduction, lequel correspond à une période bien particulière de l'histoire culturale de la région.

La reconnaissance d'une innovation renvoie aux représentations que les acteurs se font du développement (Aubertin, 1999) et du changement technique. Pour les agriculteurs de Santa Maria, andropógon est une plante momentanément idéale dans une situation donnée, mais elle ne saurait être envisagée comme une solution définitive dans une perspective de développement durable. Les pâturages, disent les gens, "c'est comme les personnes, vous voyez? On travaille, on va en vieillissant, on s'affaiblit et puis on meurt». Finalement, la durabilité prônée par les organismes de développement local, qui cherchent à stabiliser l'agriculture familiale, reste une idée assez étrangère à ceux dont l'existence est marquée de migrations successives, d'ailleurs pas nécessairement vécues comme des échecs. La mobilité (spatiale 
et sociale) des agriculteurs familiaux semble être un facteur favorable à la diffusion et à l'appropriation d'innovations. Cela dit, l'abandon d'un pâturage lorsqu'il est trop dégradé et envahi par les adventices renvoie bien aux inquiétudes que suscitent, dans un futur plus ou moins lointain, la fermeture du front pionnier, c'est-àdire l'épuisement des terres de forêt toujours nécessaires aux agriculteurs familiaux.

La nouvelle graminée a finalement été adoptée par tous les habitants de Santa Maria mais avec des motivations variables. L'analyse de la dimension sociale de l'innovation technique, notamment les modes, les rythmes et les raisons de son acceptation dans une localité donnée, montre des différences de comportement à prendre en compte dans les caractérisations de l'agriculture familiale, celles des réseaux de relation et d'échange et dans l'élaboration de programmes de développement.

\section{Remerciements}

À tous les agriculteurs de Santa Maria et leurs familles, pour leur accueil et leur patience, en particulier nos amis Fidelcino, Placidino, Claudionor, Sebastião, Mauro e Jeremias, sans qui ce travail aurait été impossible. À Annette Hladik, Catherine Grimaldi, Annie Walker, Jean-Christian Tulet et le comité de rédaction de la revue Natures Sciences Sociétés pour leurs lectures attentives du manuscrit. Le financement de ces études a été assuré par le ministère français de l'Environnement (projet SOFT), par le CNRS (projet SEAH), le CNPq, l'INPA, le LASAT et l'IRD.

Venu d'Espirito Santo à la recherche d'une vie meilleure, M. Fidelcino était fier des pâturages qu'il avait durement gagnés sur la forêt. Une longue maladie l'a d'abord amené à séjourner en ville, puis à vendre son exploitation de Santa Maria et à racheter d'autres terres dans une zone forestière éloignée. Sa famille se trouve actuellement sur ce lot, "na luta», comme il aimait à le dire. Trouvera-t-on des solutions pour que les agriculteurs puissent rester sur leurs terres, s'ils le désirent, sans avoir à migrer tous les 20 ans faute d'option?

\section{Références}

Albaladejo, C., 2001. À la recherche d'une agriculture «durable » sur les fronts pionniers : les processus de sédentarisation d'une agriculture familiale en Amazonie et en Argentine, Natures Sciences Sociétés, 9, 2, 29-43.

Alter, N., 2000. L'Innovation ordinaire, Paris, PUF, coll. «Quadrige», 2003.

Araújo, R., 1996. Fondation d'un lieu et formes de domination sur les fronts pionniers, Lusotopie, 243-252.

Arnauld de Sartre, X., 2003. Agriculture familiale en front pionnier amazonien : la sédentarisation en question, Natures Sciences Sociétés, 11, 2, 158-168.
Assis, S.W. de, 2001. Organizações sociais locais e o processo de inovações no caso da agricultura familiar ne Amazônia. Thèse de Mestrado du Núcleo de Estudos sobre Agricultura Familiar, Centro Agrário, Université fédérale du Pará, Belém, Brésil.

Aubertin, C., 1999. Développement régional et innovation. Amazonie et Centre-Ouest brésiliens, in Chaveau, J.-P., Cormier-Salem, M.-C., Mollard, E. (Eds), L'Innovation en agriculture. Questions de méthodes et terrains d'observation, Paris, IRD Éditions, 213-230.

Becker, B., 1990. Fronteira amazônica : questões sobre a gestão do território, Brasília, Editora UnB.

Buainain, A.M., Souza Filho, H.M. de, Silveira, J.M. da., 2002. Inovação tecnológica na agricultura e a agricultura familiar, in Lima, A., Wilkinson, J. (Orgs), Inovações nas tradições da agricultura familiar, Brasília, CNPq/Paralelo 15, 47-81.

Chauveau, J.-P., 1995. L'innovation cacaoyère. Histoire comparée de la cacaoculture en Afrique de l'Ouest, in Byé, P., Muchnik, J. (Eds), Innovations et sociétés. I. Dynamismes temporels de l'innovation, Montpellier, CIRAD, 43-58.

Cosenza, G.W., Andrade, R.P. de, Gomes, D.T., Rocha, C.M.C. da, 1989. Resistência de gramíneas forrageiras à cigarrinhadas-pastagens, Pesquisa Agropecuária Brasileira, 24, 8, 961968.

Darré, J.-P., 1996. L'Invention des pratiques dans l'agriculture, Paris, L'Harmattan/CNRS Éditions.

Dias Filho, M.B., 1986. Espécies forrageiras e estabelecimento de pastagens na Amazônia, in Peixoto, A.M., Moura, J.C., Faria, V.P. de (Eds), Pastagens na Amazônia, Piracicaba, FEALQ, 27-54.

Dias Filho, M.B., Serrão, E.A.S., 1980. Observações preliminares sobre a gramínea forrageira Andropogon gayanus Kunth em Paragominas, Pará, Pesquisa em andamento, Belém, EMBRAPA/CPATU, 23.

Figuié, M., 2001. A construção social dos saberes sobre a degradação dos recursos naturais nas explorações agrícolas familiares no município de Silvânia no Brasil. Thèse de doctorat, INA P-G, Paris (version en portugais).

Jones, C.A., 1979. The potencial of Andropogon gayanus Kunth in the Oxisol and Ultisol Savannas of Tropical America, Herbage Abstracts, 19, 1-8.

Klink, C.A., 1996. Germination and seedling establishment of two native and one invading African grass species in the Brazilian cerrado, Journal of Tropical Ecology, 12, 139-147.

Leite, G.C., Andrade, R.P. de, Ramos, A.K.B., Batista, L.A.R., 2001. Capim Jaraguá - Hyparrhenia rufa (Ness) Stapf. e Andropogon gayanus Kunth, in Peixoto, A.M., Pedreira, C.G.S., Moura, J.C., Faria, V.P. de (Eds), A planta forrageira no sistema de produção, Anais do $17^{\circ}$ simpósio sobre manejo de pastagem, Piracicaba, FEALQ, 225-258.

Leite, G.G., Zoby, J.L.F., 1999. Utilização integrada de pastagens de Andropogon gayanus cv. Planaltina e Brachiaria brizantha cv. Marandu, Comunicado técnico, 6, Brasília, EMBRAPACPAC.

Léna, P., 1992. Trajectoires sociales, mobilité spatiale et accumulation paysanne en Amazonie brésilienne : un exemple en Rondônia, Cahiers de sciences humaines, 28, 2, 209-234.

Léna, P., 1999. La forêt amazonienne : un enjeu politique et social contemporain, Autrepart, 9, 98-120.

Mejía, M.M., 1984. Andropogon gayanus Kunth : Bibliografia analítica, CIAT, Cali, Colombia.

Mitja, D., 1992. Influence de la culture itinérante sur la végétation d'une savane humide de Côte-d'Ivoire (Booro-Borotou - Touba), Paris, ORSTOM, coll. «Études et thèses ». 
Mitja, D., Leal Filho, N., Topall, O., 1998. Pour une réhabilitation des pâturages amazoniens dégradés, l'exemple d'Andropogon gayanus Kunth (Marabá, Pará, Brésil), Revue d'écologie (Terre Vie), 53, 39-57.

Mitja, D., 2004. A chegada do andropógon numa comunidade da Amazônia oriental : vantagens e desvantagens deste capim, multigrad, Brasília, IRD/EMBRAPA-CPAC.

Mollard, E., 1999. L'innovation est-elle risquée? Un point de vue agroéconomique, in Chauveau, J.-P., Cormier-Salem, M.-C., Mollard, E. (Eds), L'Innovation en agriculture. Questions de méthodes et terrains d'observation, Paris, IRD Éditions, 43-64.

Peixoto, A.M., Moura, J.C., Faria, V.P. de, 1995. O capim colonião, Anais do $12^{\circ}$ simpósio sobre manejo da pastagem, Piracicaba, FEALQ.

Reynal, V. de, Muchagata, M.G., Topall, O., Hebette, J., 1995. Agriculturas familiares e desenvolvimento em frente pioneira amazônica, Paris/Belém/Pointe-à-Pitre, GRET, LASAT/CAT, DAT/UAG.

Reynal, V. de, 1999. Agricultures en front pionnier amazonien, région de Marabá (Pará, Brésil). Thèse de doctorat, INA P-G, Paris.

Rogers, E.M., 1983. Diffusion of innovations, New York, The Free Press.

Roy, G., 2002. A agricultura familiar nas frentes de colonização da transamazônica : ensaio crítico sobre as abordagens agroeconômicas, Agricultura Familiar, 1, 3, 81-107.

Saraiva, M.P., Barcellos, A. de O., 1996. Desempenho animal e de pastagens de Andropogon gayanus cv. Planaltina e Brachiaria brizantha cv. Marandu submetidas a pastejo rotacionado, $2^{\circ}$ congresso de iniciação científica da UnB, Brasília, 149.

Serrão, E.A.S., Toledo, J.M., 1990. The search for sustainability in Amazon Pastures, in Anderson, A. B. (Ed.), Alternatives to deforestation: steps toward sustainable use of the amazon rain forest, New York, Colombia University Press, 195-214.

Teixeira, J.F., Simão Neto, M., Couto, W.S., Dias Filho, M.B., Silva, A.B., Duarte, M.L.R., Albuquerque, F.C., 2000. Prováveis causas da morte do capim-brachiarão (Brachiaria brizantha cv. Marandu) na Amazônia Oriental : Relatório técnico, Documentos, 36, Belém, EMBRAPA/CPATU.

Thomas, D., Andrade, R.P. de, 1984. Desempenho agronômico de cinco gramíneas tropicais sob pastejo na região dos cerrados, Pesquisa Agropecuária Brasileira, 19, 8, 1047-1051.

Thomas, D., Andrade, R.P. de, Couto, W., Rocha, C.M.C. da, Moore, P., 1981. Andropogon gayanus var. bisquamulatus cv. Planaltina : principais características forrageiras, Pesquisa Agropecuária Brasileira, 16, 3, 347-355.

Topall, O., 2001. Effet de la défoliation et des caractéristiques du milieu sur la dégradation des peuplements fourragers en région de frontière agricole amazonienne. Région de Marabá, Pará, Brésil. Thèse de doctorat, INA P-G, Paris.

Tourrand, J.F., Veiga, J.B. da, Oliveira Mares Gui, A.P. de, Carvalho, S., Oliveira Pessoa, R. de, 1995. Stratégies et pratiques d'élevage en Amazonie brésilienne. Dynamisme et diversité dans l'agriculture familiale, in Fertilité du milieu et stratégies paysannes en zone tropicale humide, Montpellier, CIRAD/AGER, 197-205.

Veiga, I., 1999. Savoirs locaux et organisation sociale de l'agriculture familiale amazonienne: la gestion durable des milieux en question. Thèse de doctorat, Université de Toulouse-le-Mirail, Toulouse.

Veiga, I., Albaladejo, C., 2002. Gestão da fertilidade dos solos de uma localidade na Amazônia Oriental, Agricultura Familiar, $1,3,109-137$.

Veiga, J.B. da, 1995. Rehabilitation of degraded pasture areas, in Management and rehabilitation of secondary forests in Amazonia, proceedings of an international Symposium/Workshop, Santarém, Pará, 193-202.

Veiga, J.B. da, Tourrand, J.-F., 2001. Pastagens cultivadas na Amazônia Brasileira : situação atual e perspectivas, Documento, 83, Belém, EMBRAPA-CPATU.

Yung, J.-M., Bosc, P.-M., Losch, B., 1995. Stratégies des producteurs et phénomènes d'innovation au Sahel, in Chauveau, J.-P., Yung, J.-M. (Eds), Innovations et sociétés. II. Les Diversités de l'innovation, Montpellier, CIRAD, 273-283.

Reçu le 28 février 2002. Accepté le 3 juin 2004.

To access this journal online: www.edpsciences.org 